\title{
Structure and transport coefficients of liquid Argon and neon using molecular dynamics simulation
}

\author{
${ }^{1}$ O. O. Odeyemi \\ ${ }^{I}$ Department of Physics, Ekiti State University, Ado-Ekiti, Ekiti State, Nigeria
}

\begin{abstract}
Molecular dynamics simulation was employed to deduce the dynamics property distribution function of Argon and Neon liquid. With the use of a Lennnard-Jones pair potential model, an inter-atomic interaction function was observed between pair of particles in a system of many particles, which indicates that the pair distribution function determines the structures of liquid Argon. This distribution effect regarding the liquid structure of Lennard-Jones potential was strongly affected such that its viscosity depends on density distribution of the model. The radial distribution function, $g(r)$ agrees well with the experimental data used. Our results regarding Argon and Neon show that their signatures are quite different at each temperature, such that their corresponding viscosity is not consistent. Two sharps turning points are more prominent in Argon, one at temperature of 83.88 Kelvin (K) with viscosity of -0.548 Pascal second (Pa-s) and the other at temperature of $215.64 \mathrm{~K}$ with viscosity of $-0.228 \mathrm{~Pa}$-s.

In Argon and Neon liquid, temperature and density are inversely and directly proportional to diffusion coefficient, in that order. This characteristic suggests that the observed non linearity could result from the non uniform thermal expansion in liquid Argon and Neon, which are between the temperature range of $21.98 \mathrm{~K}$ and $239.52 \mathrm{~K}$.
\end{abstract}

\section{Introduction}

One of the methods of computer experimentation is simulation. This simulation is deterministic in nature such that it relies on a set of initial values or condition in the estimation of intended parameter in the computation process. In classical many-body system, Frenkel and Smit (1996) reported that computation of transport properties strongly rely on simulation techniques. Several scientists like Verlet, 1962; Alder et al., 1970; Levesque et al., 1972 to mention a few have worked on the properties of matter using computational simulation. For example, Verlet (1962) used Leonard Jones model to calculate ties of liquid Argon interacting in pair-wise. Levesque (1972) studied 864 samples of Argon interacting in pair-wise; their potentials $\left(r_{c}\right)$ were cut between the range of $2.5 \sigma$ and $3.3 \sigma$. He then suggested that there is an overall agreement between the result obtained and the thermodynamic study of real Argon. $\mathrm{He}$ concluded and recommended that those results can be improved such that a two-body potential could fit into the experimental data with larger degree of interaction. Alder et al., 1970 used molecular dynamic (MD) model to investigate transparent coefficient for a hard sphere fluid. They focus their interest on the compatibility of diffusion coefficient (D), shear and thermal conductivity, which is varying. Using classical fluid, Levesque et al., 1972 investigated time auto correction function of Lennard-Jones (LJ) potential model near its triple point. They suggested that the existence of a tail extending at large times in the Kubo function defines the shear viscosity.

The MD model used by Alder et al., 1970 is one of the promising models that have revealed stronger correlation coefficients with the real experiment. As a result of this characteristic, it stands as one of the reliable numerical methods that could be used to investigate various properties of matter. One of the main reasons is that in many test-runs, its results are very similar to real experiment, that is, a sample of material that one intends to study. This can be done by connecting it to a measuring instrument, then measure the property of interest during a certain time interval, while a set of interacting atoms is followed by integrating their equations. Following the law of classical mechanics in MD simulation, where Newton's law of motion is

$$
\mathrm{F}_{i}=\mathrm{m}_{i} a_{i}
$$

For each atom $\mathrm{i}$, it is assumed that all atoms have equal mass in the system constituted by $\mathrm{N}$ atoms. Where $\mathrm{m}$ is the atomic mass, such that;

$a_{i}=d^{2} r / d t^{2}$

and $\mathrm{F}_{\mathrm{i}}$ is the force acting upon as a result of the interaction with other atoms.

Similarly, in a MD simulation, a model system that consist a number of particles (N) are solved for the Newton's equation until properties of the system are no longer changing with time, then, the system are equated. After equating the system, the actual measurement will be initiated. Therefore, observables are express as a function of positions and particles in the system measuring its quantity in MD simulation.

It is clearer from the aforementioned investigations that no work has been done on liquid Neon. In this work, structural and dynamical properties of liquid Argon and Neon, a group 8 element, will be investigated using MD simulation. The structural property is the radial distribution function $\mathrm{g}(\mathrm{r})$ and the dynamical properties are the normal temperature $(\mathrm{T})$, 
reduced temperature $\left(T^{*}\right)$, reduced density $\left(\rho^{*}\right)$, diffusion coefficient $(\mathrm{D})$, viscosity $(\eta)$ and mean-square displacement $\left(r_{\max }\right)$. These systems; Argon and Neon were chosen based on the availability of parameters that could enter the potential function and vaporization energies of the system. The $\mathrm{g}(\mathrm{r})$ is as well called the pair distributional/correlation function in the case of pair interactions. This $\mathrm{g}(\mathrm{r})$ is the most notable property among these aforementioned properties; plays significant role in determinations of theories of liquid state and effectively characterizes the local structures of fluid.

\section{Methodology}

It is easier to measure the radial distribution function $\mathrm{g}(\mathrm{r})$ in a simulation, because, it is the ratio between the average number of density $\rho(\mathrm{r})$ at a distance (r) from any given atom. In such scenario, it is assumed that all atoms are identical and the $\rho(\mathrm{r})$ at $\mathrm{r}$ from an atom in an ideal gas are the same over all densities. Structural calculations are obtained through the pair distribution function given by Frenkel and Smit (1996) as;

$$
\mathrm{g}(\mathrm{r})=\frac{\mathrm{V}}{\mathrm{N}}\left(\frac{\Delta \mathrm{N}}{4 \pi r^{2} \Delta r}\right)
$$

This is an integer over the simulation time steps at equilibrium. The volume of the box with $\mathrm{N}$ particles is $\mathrm{V}$, while $\Delta \mathrm{N}$ represent particles in a shell within the region $r-(\Delta r / 2)$ and $r+(\Delta r / 2) r$, where $\Delta r$ is the shell thickness, that is, $\Delta r$ «r. Since $r$ and $\Delta r$ are measure of position or distance, they are in units of length. The $\mathrm{g}(\mathrm{r})$ function is a time independent static equilibrium system that measures the static structure of a matter and describes spatial organization of molecules about a central molecule (Haile, 1992). The $g(r)$ function plays a key role in the pair distribution function of dense fluids and provides a signature for identifying the lattice structure of crystalline solids. The probability to find a pair, which is a distance $r$ apart, relative to what is expected for a uniform random distribution at the same density is define by Haile (1992) as;

$$
\begin{gathered}
\rho g(\mathrm{r})=\frac{1}{\mathrm{~N}}\left\{\sum_{i=1}^{n} \sum_{j=1}^{n}\left(\mathrm{r}-\mathrm{r}_{\mathrm{ij}}\right)\right\} \\
\mathrm{j} \neq \mathrm{i}
\end{gathered}
$$

The angular bracket is the time average, $\rho$ is the density number, $\mathrm{N}$ is the total number of particles and $\mathrm{r}_{\mathrm{ij}}$ is the vector between centers of atoms $\mathrm{i}$ and $\mathrm{j}$. The average number of atoms located between $r_{i}$ and $r_{j}$ from a given atom is given by the expression;

$$
\rho \int_{r} g(r) 4 \pi \mathrm{r}^{2} d r
$$

This allows defining the coordination number when disorder occurs.

Diffusion coefficient (D) and viscosity ( $\eta$ ) are some of the dynamical properties that will be consider in this paper. Diffusion is a transport process or dynamical phenomenon that results from molecular motion of particles in fluids. The Fick's law is a microscopic law, which describes $\mathrm{D}$ and states that the flux $(\mathrm{J})$ of the diffusing species is directly proportional to the negative gradient $(\nabla)$ in the concentration $(C)$ of species. That is,

$$
\mathrm{J}=-\mathrm{D} \nabla \mathrm{C}
$$

Where, $\mathrm{D}$ is the constant of proportionality.

Unlike D, n was obtained through a well-known Kubo-like formula of

$$
\eta=\int_{0}^{\infty} \eta(t) d t
$$

with

$$
\eta(\mathrm{t})=\frac{\rho}{3 K_{B} T} \sum_{G}\left(\frac{T^{X Y}(0) \mathrm{T}^{X Y}(\mathrm{t})}{N}\right)
$$

Where, $\mathrm{T}^{\mathrm{XY}}$ is the shear stress, the sum is made on the circular permutation of indices and the bracket implies that the average is divided over the (initial) time.

These properties are considered in an ensemble, such that the number of particles $\mathrm{N}$, the Volume $\mathrm{V}$ and the total energy $\mathrm{E}$ of the 
system are constants. These indicate that a micro-canonical ensemble, which is an isolated system, does not interact with the rest of the universe or its environment. Hence, it is characterized by the state $\mathrm{N}, \mathrm{V}$ and $\mathrm{E}$.

Lennard-Jone (LJ) potential model is employed to compute this isolated system. One of the reasons is that it describes the interatomic functions between pair of particles in a system of many particles. Given by the expression

$$
\mathrm{V}(\mathrm{r})=\mathrm{N}(\mathrm{N}-1)
$$

$$
\sum \sum 4 \in\left[\left(\frac{\sigma}{r}\right)^{12}-\left(\frac{\sigma}{r}\right)^{6}\right]
$$

The term $\left(\frac{\sigma}{r}\right)^{12}$ is a repulsive term that accounts for the columbic or screening effect of atoms pair. This repulsive term effect creates a short range order, which is a characteristic of the liquid state. The range of this repulsion is roughly equal to the nearest neighbor distance. The term $\left(\frac{\sigma}{r}\right)^{6}$ is an attractive term, which arises from Van der waals forces. These attractive forces bind the atoms together at long range and vary much more smoothly with the distance between particles. It also plays a minor role in determining the structure of the liquid (Verlet, 1962).

Before triggering the simulation, the particles positions and velocities are initialized. This initialization of particles positions and velocities must be strictly compatible with intending structure the investigator is aiming to simulate (Frenkel and Smit, 1996). For example, the particles should not be positioned in such a way that results to overlapping of atomic or molecular cores. Recall that in thermal equilibrium,

$$
\left(\mathrm{V}_{\alpha}\right)=\frac{\mathrm{K}_{\mathrm{B}} \mathrm{T}}{\mathrm{m}}
$$

Where $\left(\mathrm{V}_{\alpha}\right)$ is the component $\alpha$ of the velocity of a given particle, we define as instantaneous temperature at time $\mathrm{t}(\mathrm{t})$ through this relation

$$
\mathrm{K}_{\mathrm{B}} \mathrm{T}(\mathrm{t}) \cong \sum_{i=1}^{\mathrm{N}} \frac{\mathrm{mV}_{\alpha_{i}}^{2}(\mathrm{t})}{\mathrm{Nf}}
$$

$\mathrm{m}$ is Brownian particle mass

$\mathrm{N}$ is number of particles

$\mathrm{K}_{\mathrm{B}}$ is Boltzmann constant

During equilibrium, the temperature will adjust itself because, it is not critical.

The most time consuming part of MD simulation the calculation of force acting on every particle. Considering LJ potential with pair wise additive interaction with contribution of particle i due to its neighbor, interaction only between a particle and the nearest image of another particle as well, then, for a system of $\mathrm{N}$ particles, pair distance $\frac{N(N-1)}{2}$ is evaluate.

If given pair of particles are closer to interact, forces of particles and the contribution of the potential energy are easier to calculate. For example, if one wants to x-component of this force,

$$
\begin{gathered}
\operatorname{Fx}(\mathrm{r})=-\frac{\partial \phi(\mathrm{r})}{\partial x} \\
=-\frac{\left(\frac{x}{r}\right) \partial \phi(r)}{\partial x}
\end{gathered}
$$

then for a $\mathrm{LJ}$ system in a reduced unit

$$
\operatorname{Fx}(\mathrm{r})=\frac{48 X}{r^{2}\left[\frac{1}{\mathrm{r}^{12}}-\frac{0.51}{\mathrm{r}^{6}}\right]}
$$

The Verlet and predictor-corrector algorithms are most popularly used MD simulations. To derive the algorithm, we start with the Taylor expansion of the coordinate of a particle around time, $t$ (Furio, 1997), where

$$
\mathrm{r}(\mathrm{t}+\Delta \mathrm{t})=\mathrm{r}(\mathrm{t})+\mathrm{v}(\mathrm{t}) \Delta \mathrm{t}+\frac{1}{2} a(t) \Delta \mathrm{t}^{2}+\frac{1}{6} b(t) \Delta \mathrm{t}^{3}+0\left(\Delta \mathrm{t}^{4}\right)
$$


Where $t$ is the force divided by mass and the force is a function of $r(t)$ position

$a(t)=-\frac{1}{m} \nabla V[r(t)]$

and $\mathrm{b}$ is the $3^{r d}$ derivative of the position $\mathrm{r}(\mathrm{t}), \frac{\mathrm{d}^{3} r(t)}{\mathrm{d} t^{3}}$ wrt $\mathrm{t}$

$\mathrm{r}(\mathrm{t}+\Delta \mathrm{t})=\mathrm{r}(\mathrm{t})+\mathrm{v}(\mathrm{t}) \Delta \mathrm{t}+\frac{1}{2} a(t) \Delta \mathrm{t}^{2}+\frac{1}{6} b(t) \Delta \mathrm{t}^{3}+0\left(\Delta \mathrm{t}^{4}\right)$

adding these two expressions we have,

$\mathrm{r}(\mathrm{t}-\Delta \mathrm{t})=2 \mathrm{r}(\mathrm{t})-\mathrm{r}(\mathrm{t}-\Delta \mathrm{t})+a(t) \Delta \mathrm{t}^{2}+0\left(\Delta \mathrm{t}^{4}\right)$

The truncating error associated with equations (17) is of the order of $\Delta t^{2}$, however, the third derivative cancels out and the fourth derivative is zero. One of the main disadvantages of this algorithm (equation 17) is that, it cannot be generated directly. Then, velocities can be computed from positions by

$$
V(t)=(t+\Delta t)-\frac{r(t+\Delta t)}{2 \Delta t}
$$

Development of velocity Verlet algorithm (VVA) was able to overcome this truncating error associated with equations 17, which is of the order of $\Delta t^{2}$. The estimation of positions, velocities and accelerations using VVA at time $t+\Delta t$ are obtained from the same quantity at time $t$ as follow;

$$
\begin{aligned}
& \mathrm{r}(\mathrm{t}+\Delta \mathrm{t})=\mathrm{r}(\mathrm{t})+\mathrm{r}(\mathrm{t}) \Delta \mathrm{t}+\frac{1}{2} a(\mathrm{t}) \Delta \mathrm{t}^{2} \\
& \mathrm{v}(\mathrm{t}+\Delta \mathrm{t})=\mathrm{v}(\mathrm{t}) \Delta \mathrm{t}+\frac{1}{2} a(t) \Delta \mathrm{t} \\
& a(\mathrm{t}+\Delta \mathrm{t})=-\frac{1}{m} \nabla[r(\mathrm{t}+\Delta \mathrm{t})] \\
& \mathrm{v}(\mathrm{t}+\Delta \mathrm{t})=\mathrm{v}\left(\mathrm{t}+\frac{\Delta \mathrm{t}}{2}\right)+\frac{1}{2} a(\mathrm{t}+\Delta \mathrm{t}) \Delta \mathrm{t}
\end{aligned}
$$

On the predictor corrector part of VVA, we adopt compromise between accuracy and stability. The idea is to use the information about the first $\mathrm{n}$ derivatives at time $\mathrm{t}$ to arrive at a prediction for a position and its first derivative at time $t+\Delta t$. Therefore, computation of the force and acceleration at the predicted position could be easier to achieve. If there is discrepancy between predicted and observed accelerations, our estimates of positions and the remaining (n-1) derivatives could be improve. For example, considering Taylor expansion of the coordinate of a given particle at time $t+\Delta t$ as follow;

$$
\begin{aligned}
& \mathrm{r}(\mathrm{t}+\Delta \mathrm{t})=\mathrm{r}(\mathrm{t})+\Delta \mathrm{t} \frac{\delta r}{\delta t}+\frac{\Delta \mathrm{t}^{2}}{2 !} \frac{\delta^{2} r}{\delta t^{2}}+\frac{\Delta \mathrm{t}^{3}}{3 !} \frac{\delta^{3} r}{\delta t^{3}}+\ldots \ldots . . \\
& \mathrm{X}_{0}(\mathrm{t}) \cong \mathrm{r}(\mathrm{t}) \\
& \mathrm{X}_{1}(\mathrm{t}) \cong \Delta \mathrm{t} \frac{\delta r}{\delta t} \\
& \mathrm{X}_{2}(\mathrm{t}) \cong \frac{\Delta \mathrm{t}^{2}}{2 !} \frac{\delta^{2} r}{\delta t^{2}} \\
& \mathrm{X}_{3}(\mathrm{t}) \cong \frac{\Delta \mathrm{t}^{3}}{3 !} \frac{\delta^{3} r}{\delta t^{3}}
\end{aligned}
$$

We can write the following predictions for $\mathrm{X}_{0}(\mathrm{t}+\Delta \mathrm{t})$ through to $\mathrm{X}_{3}(\mathrm{t}+\Delta \mathrm{t})$

$$
\mathrm{X}_{0}(\mathrm{t}+\Delta \mathrm{t})=\mathrm{X}_{0}(\mathrm{t})+\mathrm{X}_{1}(\mathrm{t})+\mathrm{X}_{2}(\mathrm{t})+\mathrm{X}_{3}(\mathrm{t})
$$




$$
\begin{array}{lr}
X_{1}(t+\Delta t)= & X_{1}(t)+X_{2}(t)+X_{3}(t) \\
X_{2}(t+\Delta t)= & X_{2}(t)+X_{3}(t) \\
X_{3}(t+\Delta t)= & X_{3}(t)
\end{array}
$$

Since we have $X_{0}(t)$, we can compute forces at predicted positions and as well compute the corrected values for $X_{2}(t+\Delta t)$. To do that, denote the difference between

$\mathrm{X}_{2}^{\text {correct }}$ and $\mathrm{X}_{2}^{\text {predicted }}$ by $\Delta \mathrm{X}_{2} \Delta \mathrm{X}_{2}=\mathrm{X}_{2}^{\text {correct }}-\mathrm{X}_{2}^{\text {predicted }}$

then, we estimate corrected values of $X_{0}$ through to $X_{3}$ as follows;

$\mathrm{X}_{n}^{\text {corrected }}=\mathrm{X}_{n}^{\text {predicted }}+\mathrm{C}_{n} \Delta \mathrm{X}_{2}$

(32) Where, the $C_{n}$ are constants that are fixed for a given order of algorithm. All corrections are proportional to signal error signal such that values of $\mathrm{Cn}$ yield optimal compromise between the stability and accuracy of the algorithm. In a MD simulation, the D can as well be determined from the mean square displacement (MSD). The equation

$2 \mathrm{D}=\lim _{t \rightarrow \infty} \frac{\partial\left[x^{2}(t)\right]}{\partial t}$

shows that $\mathrm{D}$ is the gradient of MSD. In this case, only one Cartesian of the mean square displacement is considered. This equation is valued within the limit of $t \rightarrow \infty$. In practice, it verification have to be in-place to confirmed that the simulation is adequate. The overall result is that the MSD is directly proportional to $t$ and not another power of $t$. Therefore, normal temperature $(\mathrm{T})$, reduced temperature $\left(T^{*}\right)$, reduced density $\left(\rho^{*}\right)$, diffusion coefficient $(\mathrm{D})$, viscosity $(\eta)$ and mean square displacement $\left(r_{\text {max }}\right)$ are simulated from MD model.

III. Results

Table 1: Calculated structural properties from diffusion coefficient (D) of atom in liquid Argon

\begin{tabular}{lllllll}
\hline \hline System & $\mathrm{T}$ & $\mathrm{T}^{*}$ & $\rho^{*}$ & $\mathbf{D}$ & $\eta$ & ${ }^{\mathrm{r}}{ }_{\max }$ \\
\hline \hline Argon & 71.88 & 0.599 & 0.950 & 0.4490 & 4.656 & 0.9848 \\
Argon & 77.88 & 0.649 & 0.900 & 0.3868 & 1.965 & 1.0026 \\
Argon & 83.88 & 0.699 & 0.850 & 0.3255 & -0.548 & 1.0219 \\
Argon & 95.88 & 0.799 & 0.600 & 0.0893 & -0.088 & 1.0908 \\
Argon & 107.76 & 0.898 & 0.550 & 0.0957 & 0.006 & 1.1034 \\
Argon & 119.76 & 0.998 & 0.550 & 0.1052 & 0.090 & 1.0838 \\
Argon & 179.64 & 1.497 & 0.450 & 0.2440 & 0.202 & 1.0962 \\
Argon & 215.64 & 1.797 & 0.400 & 0.3796 & -0.228 & 1.0966 \\
Argon & 239.52 & 1.996 & 0.350 & 0.5573 & 0.359 & 1.1011 \\
\hline \hline
\end{tabular}

Where T is the normal temperature, $T^{*}$ is the reduced temperature, $\rho^{*}$ is the reduced density, $\mathrm{D}$ is the diffusion coefficient, $\eta$ viscosity and $r_{\max }$ is the mean square displacement.

Table 2: Calculated structural properties from diffusion coefficient (D) of atom in liquid Neon

\begin{tabular}{lcccccc}
\hline \hline System & $\mathrm{T}$ & $\mathrm{T}^{*}$ & $\rho^{*}$ & $\mathbf{D}$ & $\eta$ & ${ }^{\mathrm{r}}{ }_{\max }$ \\
\hline \hline Neon & 21.98 & 0.599 & 0.950 & 0.4490 & 4.656 & 0.9848 \\
Neon & 23.82 & 0.649 & 0.900 & 0.3868 & 1.965 & 1.0026 \\
Neon & 25.65 & 0.699 & 0.850 & 0.3255 & -0.548 & 1.0219 \\
Neon & 29.32 & 0.799 & 0.600 & 0.0893 & -0.088 & 1.0908 \\
Neon & 32.96 & 0.898 & 0.550 & 0.0957 & 0.006 & 1.1034 \\
Neon & 36.63 & 0.998 & 0.550 & 0.1052 & 0.090 & 1.0838 \\
Neon & 54.94 & 1.497 & 0.450 & 0.2440 & 0.202 & 1.0962 \\
Neon & 65.95 & 1.797 & 0.400 & 0.3796 & -0.228 & 1.0966 \\
\hline
\end{tabular}




\begin{tabular}{|c|c|c|c|c|c|c|}
\hline Neon & 73.25 & 1.996 & 0.350 & 0.5573 & 0.359 & 1.1011 \\
\hline
\end{tabular}

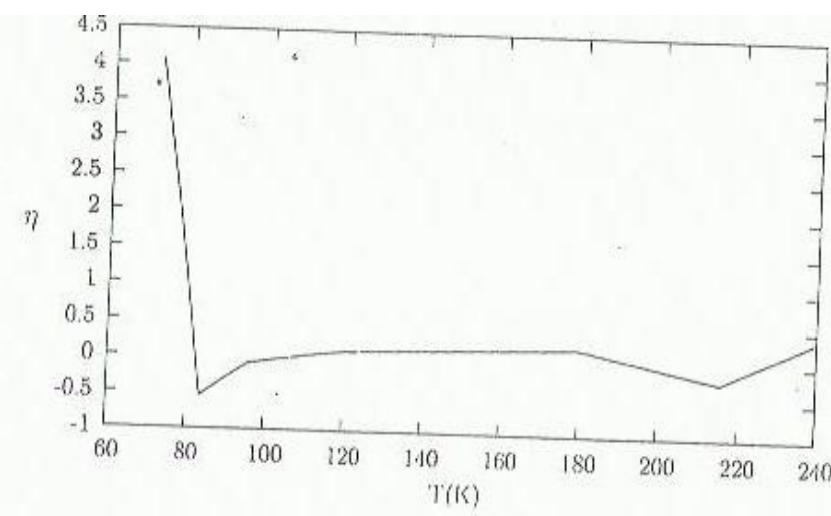

Figure 1: Variability of viscosity (in Pa-s) with temperature in liquid Argon.

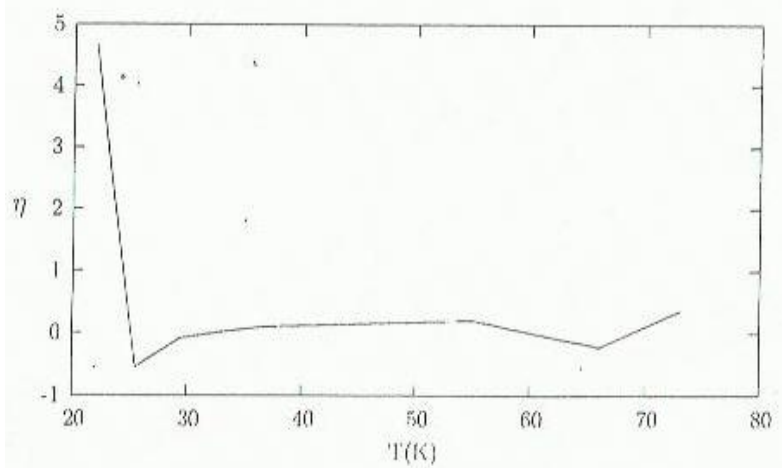

Figure 2: Variability of viscosity (Pa-s) with temperature in liquid Neon.

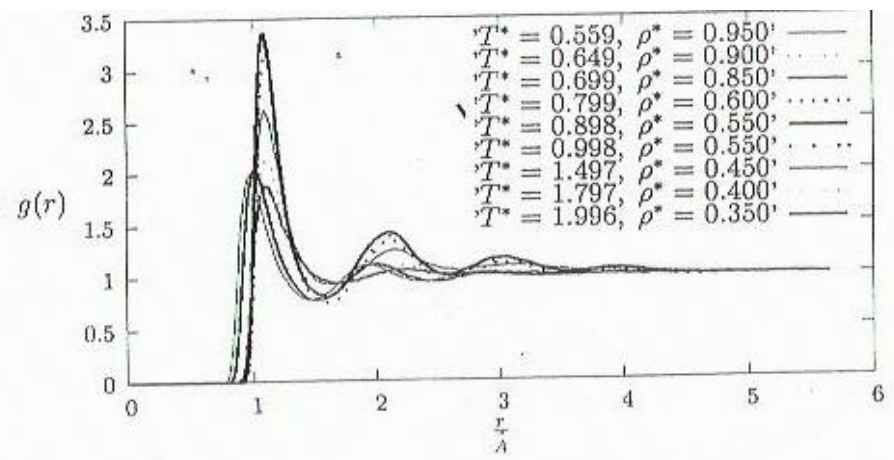

Figure 3: The pair distribution function, $\mathrm{g}(\mathrm{r})$ of Argon at reduced temperatures $(\mathrm{K})$ and densities $\left(\mathrm{Kg} / \mathrm{m}^{3}\right)$. 


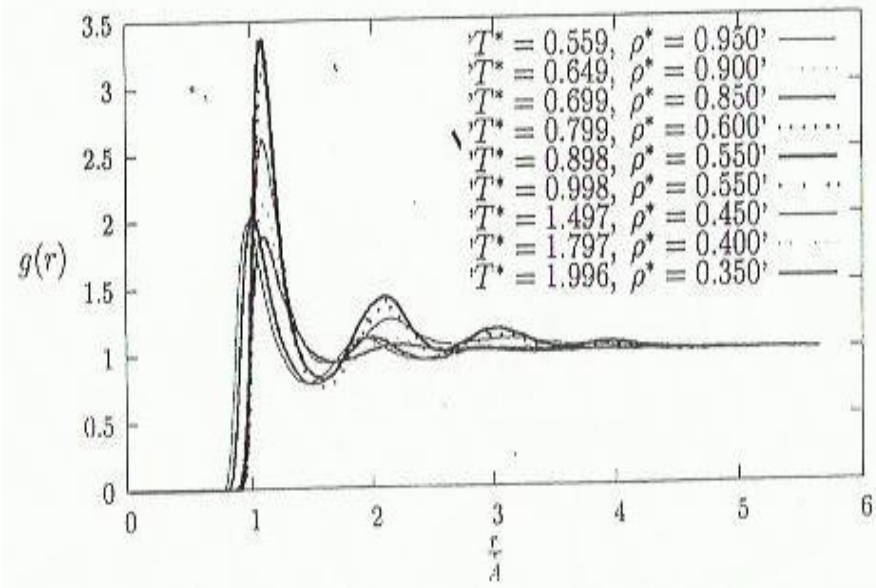

Figure 4: The pair distribution function, $\mathrm{g}(\mathrm{r})$ of Neon at reduced temperatures $(\mathrm{K})$ and densities $\left(\mathrm{Kg} / \mathrm{m}^{3}\right)$.

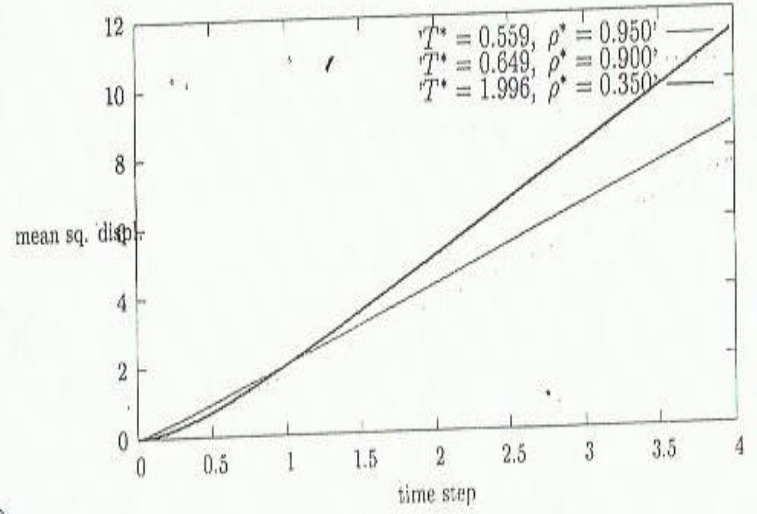

Figure 5: Variability of the mean square displacement $\left(r_{\text {max }}\right)$ of liquid Argon.

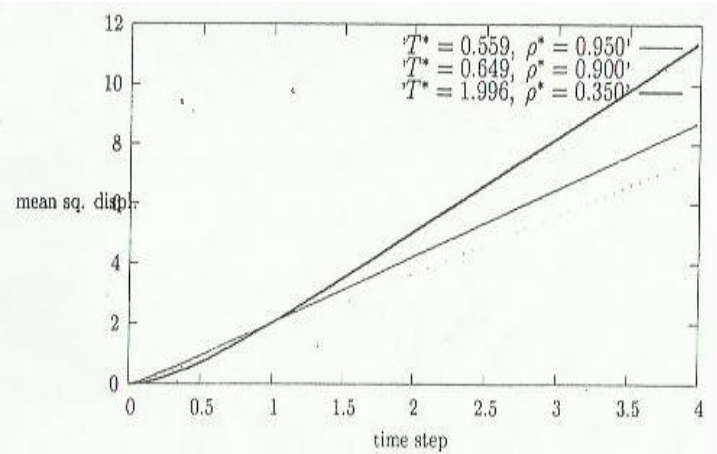

Figure 6: Variability of the mean square displacement $\left(r_{\max }\right)$ of liquid Neon.

\section{Discussions Of Results}

Results shown in table 1 and 2 were obtained using molecular dynamic (MD) model described under methodology. Apart from the pair distribution functions, $g(r)$, structural properties of liquid Argon and Neon; viscosity $(n)$ with other input parameters like normal temperature $(\mathrm{T})$, reduced temperature $\left(T^{*}\right)$, reduced density $\left(\rho^{*}\right)$, and mean square displacement $\left(r_{\max }\right)$ that are deduced from diffusion coefficient (D) are depicted with table 1 and 2 , respectively.

From Figure 1, the shear $\eta$ of Argon variability with temperature is not linear. The shear $\eta$ of liquid Argon was highest below $80 \mathrm{~K}$ and has a value of $\sim 4 \mathrm{~Pa}-\mathrm{s}$, closer to $80 \mathrm{~K}$, liquid Argon shear $\eta$ value reduces sharply and faster such that it get to below zero Pa-s around $80 \mathrm{~K}$. Closer to $90 \mathrm{~K}$, it increases slightly to around $0.1 \mathrm{~Pa}-\mathrm{s}$ and fluctuate between 0.1 $\mathrm{Pa}-\mathrm{s}$ and a little higher $(0.15 \mathrm{~Pa}-\mathrm{s})$ around $100 \mathrm{~K}$ and $180 \mathrm{~K}$. Immediately after $180 \mathrm{~K}$, liquid Argon shear $\eta$ reduces slightly and gradual to around $220 \mathrm{~K}$ and a value of $\sim-0.2 \mathrm{~Pa}-\mathrm{s}$ and later increases sharply to $\sim 0.5 \mathrm{~Pa}-\mathrm{s}$ at $240 \mathrm{~K}$. Although, similar signature closer to that of figure 1 was observed in figure 2. However, in liquid Neon, reduced temperature and increased shear $\eta$ were observed compare to shear $\eta$ of liquid Argon. Around $23 \mathrm{~K}$, highest shear $\eta$ of $~$ 4.8 Pa-s was observed in liquid Neon, which immediately decreases sharply to $\sim-0.5 \mathrm{~Pa}-\mathrm{s}$ around $25 \mathrm{~K}$. Shear $\eta$ of liquid Neon was observed increasing gradually immediately after $25 \mathrm{~K}$ and has a value of $\sim 0.1 \mathrm{~Pa}-\mathrm{s}$ at $30 \mathrm{~K}$. This value $(\sim 0.1$ 
Pa-s) was observed increasing slightly after $30 \mathrm{~K}$ and get to a maximum around $55 \mathrm{~K}$ with a value of $\sim 0.5 \mathrm{~Pa}-\mathrm{s}$. Immediately after $55 \mathrm{~K}$, sharp decrease, which is slighter, was observed and gets to a minimum value of $\sim-0.2 \mathrm{~Pa}-\mathrm{s}$ around $66 \mathrm{~K}$. Immediately after $66 \mathrm{~K}$, a sharp increase that gets beyond $70 \mathrm{~K}$ was observed. This sharp increment that was observed before $70 \mathrm{~K}$ and beyond indicates that the variability of shear $\eta$ of liquid Argon is a continuation of liquid Neon with respect to temperature. Therefore, liquid Neon shear $\eta$ could extinct at temperature above $70 \mathrm{~K}$ and above $70 \mathrm{~K}$, liquid Argon shear $\eta$ thrives. From both figures, it is clearer that the higher the temperature, the lower the shear $\eta$.

From figures 3 and 4 , the variability of $g(r)$ at reduced temperatures and densities of liquid Argon and Neon were shown, in that order. Interestingly, it was observed that this variability from both figures is the same in value. That is, they have reduced temperature $\left(T^{*}\right)$ in the range of $0.559 \mathrm{~K}$ to $1.996 \mathrm{~K}$ and reduced density $\left(\rho^{*}\right)$ in the range of 0.950 $\mathrm{Kg} / \mathrm{m}^{3}$ to $0.350 \mathrm{Kg} / \mathrm{m}^{3}$. This is the reason for the same variability shown in figure 3 and 4 , having the same peaks and position, which indicates that the structure of liquid Argon and Neon does not change with reduced $T^{*}$ and $\rho^{*}$. At $T^{*}$ of 0.799 $\mathrm{K}$ to $1.497 \mathrm{~K}$ and $\rho^{*}$ of $0.450 \mathrm{~Pa}-\mathrm{s}$ to $0.600 \mathrm{~Pa}-\mathrm{s}$ of liquid Argon Neon, the plots are similar such that there is an abrupt change in the structure of real Argon and Neon system. Also, at $T^{*}$ between $1.787 \mathrm{~K}$ and $1.996 \mathrm{~K}$ and at $\rho^{*}$ between $0.350 \mathrm{~K}$ and $0.400 \mathrm{~K}$ in liquid Argon and Neon, they have similar structure. This range indicates that the structure is more of gases than liquid because structures of gases do not oscillate, that is, liquid Argon and Neon are closer to gaseous state. Irrespective of these aforementioned characteristics, it was observed that as the $T^{*}$ increased, the $\rho^{*}$ was observed decreasing for liquid Argon and Neon. This implies that the coordination number is lower in the liquid system.

The mean square displacement $\left(r_{\text {max }}\right)$ variability was depicted in figures 5 and 6 for Argon and Neon, respectively. The values from the MD simulation that is the same reproduced the same polts for figures 5 and 6 . From both figures (5 and 6), the $r_{\text {max }}$ variability was observed increasing linearly with respect to $T^{*}$ and $\rho^{*}$ over time step. The $T^{*}$ was observed to increase from $0.559 \mathrm{~K}$ to $1.996 \mathrm{~K}$ and the $\rho^{*}$ was observed to reduce from $0.950 \mathrm{Kg} / \mathrm{m}^{3}$ to $0.350 \mathrm{Kg} / \mathrm{m}^{3}$, in that order. This implies that as $T^{*}$ is increasing, $\rho^{*}$ is reducing, which result to the linear variability observed in both figures.

\section{Conclusions}

We have investigated the dependence of the structural properties and diffusion coefficient above the melting point of liquid Argon and Neon on reduced temperature and density. Our deductions are as follows: having compared our results on structure with that of experiments, we observed that the results agree quite well and it is within the limit of experimental errors. Hence we can easily infer that, molecular dynamics simulation is very useful and reliable tool for carrying out structural studies of group 8 elements. We also inferred that, both methods of simulation, i.e. MD and Monte-Carlo methods could be used as scientific tools in Physics in order to increase our understanding as well as give insight into the Physics governing systems interaction and properties. Other potential models should also be encouraged to study this kind of work apart from Lennard-Jones fluids.

The effects of densities and temperatures on the structure, diffusion coefficient, and viscosity of systems modeled via the Lennard-Jones potential have been investigated; we therefore conclude that at temperatures above melting, the structure of Lennard-Jones liquids is affected by the temperature. Before we take reading in any simulation, we have to wait for a very long time. This is required to allow a system to attain equilibrium so that all readings taken are at equilibrium condition. The readings that are taken at non equilibrium cannot represent actual static structure and it would be wrong to use such data to calculate other properties. As a test, the structural and dynamical properties of Argon at various temperatures at reduced units were computed and compared with that of Daan Frenkel and B. Smit [4]. The agreement of our results and that of Frenkel and Smit is an indication that our chosen choice of simulation program is accurate; and that one should be able to extend the program to study other systems, which we were able to achieve, provided that the parameters of our pair potential were chosen from the literature. The parameters were calculated based on structures and energies of the systems.

\section{References}

[1] Furio (1997): Molecular dynamics primer

[2] Loup Verlet (1962): Computer experiments on classical fluids (i). Thermodynamics properties of Lennard-Jones molecules. Phys Rev 159 (1996)

[3] J. Alder, D.M Gs and T.E Wainwright (1970): Studies in molecular dynamics (vii). The transport coefficient for hard sphere fluid. Lawrence radiation laboratory, Califonia, USA.

[4] Dean Frenkel and Berend Smit (1996): Understanding molecular dynamics simulation from algorithm to applications. Published by academics press, San Diego Hondon Briston, New York.

[5] D. Levesque, L. Verlet and Juhani Kurkijarvi (1972): Computer Experiments on classical fluids iv. transport properties and time correlation functions of the Lennard-Jones liquid near its triple point, France.

[6] Jean B. Marion (1970): Classical dynamics of particles and system second edition. Published by academic press, Harcourt Brace New York.

[7] A summer Field (1950): Mechanics. Published by academic press, New York.

[8] J.M. Haile (1992): Molecular dynamics simulation elementary methods. John Wiley and Sons, Inc, USA. 